\title{
How much help does a vaccine-induced T-cell response need?
}

\author{
Jeffrey S. Weber ${ }^{1}$ and James J. Mulé ${ }^{2}$ \\ ${ }^{1}$ Department of Medicine, Keck/University of Southern California School of Medicine and Norris Comprehensive Cancer Center, \\ Los Angeles, California, USA \\ ${ }^{2}$ University of Michigan Medical Center, Department of Surgery, Ann Arbor, Michigan, USA
}

Address correspondence to: James J. Mulé, University of Michigan Medical Center, Department of Surgery, 1520C MSRBI, Box 0666, Ann Arbor, Michigan 48109-0666, USA. Phone: (734) 647-2779; Fax: (734) 763-4135; E-mail: jimmule@umich.edu.

Currently, much attention is focused on the development of tumor vaccines that incorporate defined tumor-associated antigen (TAA) peptides. Following the first report of the successful molecular cloning of a gene encoding a cytotoxic $\mathrm{T}$ lymphocyte-defined (CTLdefined) TAA (1), critical questions have surfaced - not only with respect to the optimal composition of such vaccines (including the choice of TAA, adjuvant, and delivery mode), but also how its effectiveness should be best measured in terms of laboratory and clinical parameters. There is much controversy surrounding these issues, ranging from whether a particular peptide sequence is truly a tumor rejection antigen to whether a dendritic cell, rather than some particular cytokine or nonspecific agent, would serve as the most efficient adjuvant. In a recent issue of the JCI, Knutson and colleagues describe the immunologic monitoring results of a pilot clinical trial of a multipeptide vaccine admixed with GM-CSF in patients with breast cancer (2). Surprisingly, the authors found that MHC class I-restricted epitope sequences nested inside $\mathrm{MHC}$ class II sequences were capable of eliciting CTL immunity.

This work, which incorporated a number of correlative studies measuring immune responses, was aimed in part to test the hypothesis that the direct addition of GM-CSF can boost the generation of local antigen-presenting cells, an idea that has been demonstrated in mice using GMCSF-transfected cell vaccines (3). Clinically, in small pilot studies conducted by Jager (4) and Jaffee (5) and their colleagues, the addition of GM-CSF appeared to augment antigen-specific CTL responses to peptide- or whole-cell vaccines. In contrast, recently presented preliminary data suggest that GM-
CSF does not add to the immunogenicity of a peptide vaccine prepared with incomplete Freund's Adjuvant (6). Otherwise, no randomized trials testing the clinical or immunologic utility of GM-CSF as an adjuvant in cancer patients have yet been reported. The current study (2) was also intended to test the idea that MHC class II-restricted helper peptides could generate detectable and durable $\mathrm{CD}^{+}{ }^{+} \mathrm{T}$-cell immunity in cancer patients, which would result in augmented MHC class I-restricted $\mathrm{CD}^{+} \mathrm{CTL}$ responses. The authors got an unexpected bonus, since they not only detected helper peptide-specific responses, but they also found that certain MHC class I epitopes that acted on $\mathrm{CD}^{+} \mathrm{CTL}$ correspond to sequences nested within longer helper peptides that act on $\mathrm{CD}^{+}$cells. The authors' previous small pilot trials $(7,8)$ demonstrated that breast cancer patients immunized with HER-2/neu helper peptides develop both HER-2/neu peptide-specific T-cell responses and also HER-2/neu protein-specific responses, a consequence of epitope spreading. Immune $\mathrm{T}$ cells elicited by vaccination generated delayed-type hypersensitivity (DTH) responses, which indicated the potential ability to traffic distally from the vaccine site.

\section{Dendritic cells and other adjuvants}

Recently, human studies by Bhardwaj's group have suggested that the use of mature (as opposed to immature) dendritic cells can substitute for helper epitopes to generate CTL responses to defined peptide epitopes (9), raising some doubts about the need for additional helper activity. In the present study (2), most patients developed Th responses against HER-2/neu, but it should be noted that the reactivity to this peptide was not equivalent to that seen with strong immunogens such as influenza peptide or tetanus. The use of potent adjuvants to boost immune responses would therefore be warranted in future studies of helper peptides. There are other, methodological concerns as well. To register immune responses, the authors used a sensitive ELISPOT assay, which measures restimulated cells and may not reflect quantitative aspects of the immune response as faithfully as measuring precursor Th cells or CTLs from fresh blood - or, perhaps better, from the tumor or DTH site. Antibodies against HER-2/neu were not measured in this study, which would provide further information on the biologic activity of Th responses. Finally, it is not clear whether the ELISPOT assay offers as accurate a view of tumor-specific immune responses as other assays, such as the real-time RT-PCR approach used recently in studies by Marincola and colleagues (10).

In the present study (2), the frequency of tumor antigen-specific T-cell precursors detected after helper peptide vaccination was in the range of 1:10,000, similar to memory responses seen in viral infections, but likely well below what would be considered an "acute" response required to clear a viral infection. An important question that must be answered is whether immune responses in the Knutson et al. study correlate with clinical parameters such as tumor response in patients with metastatic disease, or relapse-free or overall survival in those treated in the adjuvant setting.

The oft-expressed hypothesis that addition of helper sequences is necessary to induce a long-lived immune response has not been borne out by human data. In trials using peptide vaccines prepared with incomplete Freund's Adjuvant (11), nonspecific helper 
activity may be induced by the potent adjuvant. Similarly, in the experiments cited above by Bhardwaj and colleagues (9), the injection of ex vivo-generated mature dendritic cells obviated the need for helper antigen. A number of preclinical vaccine studies using peptides, proteins, or antigen-pulsed dendritic cells have employed nonspecific Th sequences to stimulate Th cells and to promote an augmented antibody or CTL response (12-14). Clinical studies with peptide vaccines have not confirmed that nonspecific helper peptides boost CTL immunity (15), but several recent studies indicate that nonspecific helper sequences pulsed onto dendritic cells generate strong $\mathrm{Th}$ activity $(14,16)$.

\section{Studies for the future}

It remains to be shown in humans whether the presence of helper sequences derived from an antigen containing CTL epitopes (as in the current study) is superior to the addition of nonspecific helper sequences. Indeed, it is not clear whether the epitope-specific CTL responses seen occurred because of the presence of the helper sequences covalently linked to them, or in spite of them. Only a comparison trial, using the eight to ten amino acid CTL epitopes alone without covalently linked helper sequences or the CTL epitopes admixed with nonspecific helper sequences will yield an answer. Fortunately, the current HER-2/neu system offers an ideal setting for such studies. Recent experiments have identified MHC class II-restricted helper epitopes encoded by multiple cancer antigens $(17,18)$ and should allow for more studies to test the benefit of T-cell help in augmenting CTL immunity.

The results of the current trial also indirectly test the emerging concept in cancer immunotherapy that immune suppression influences effector $\mathrm{T}$ cells (19) and antigen-presenting dendritic cells. Gabrilovitch et al. have conducted a series of experiments demonstrating that dendritic cells in breast cancer patients are decreased both in number and function (20). Assuming that peptide-based strategies require host-derived dendritic cells, the results of Knutson and colleagues suggest that the suppression of dendritic cell-dependent responses can be overcome by the authors' vaccination strategy. If dendritic cell defects could be reversed, the levels of immune response achieved could be much greater. Future vaccine trials must include strategies to measure and perhaps overcome host immune suppression.

Finally, the time has come to settle at least one controversy in this field and test the idea that helper and cytolytic epitope peptides will augment immunity to breast cancer in a clinically meaningful way. A logical approach would be to follow patients with minimal residual disease. Since virtually all women with node-positive breast cancer who are at a high risk of relapse receive adjuvant chemotherapy, any vaccine trial for breast cancer would presumably begin after myelosuppressive chemotherapy, a setting that is immunologically poorly defined. Patients who are HER-2/neu positive might also receive HER-2/neu antibody therapy, further complicating the analysis of any vaccine study in this population. A reasonable alternative would be to perform several small studies to assess whether a timing of administration of a HER-2/neu peptide vaccine relative to chemotherapy affects the strength of the immune response, and to determine whether helper epitopes are more potent when covalently linked or admixed in the vaccine. The timing and number of treatments will also need to be studied to optimize the immunological responses to the vaccine. With these preliminary data in hand, a phase III randomized trial could then be performed, testing HER2/neu peptides with adjuvant (GM-CSF or other cytokines), as compared with a placebo, and using time to relapse and overall survival as the primary clinical endpoints. A secondary endpoint would be to correlate immune response with survival - the ultimate challenge to the cancer vaccine field. The paper by Knutson and colleagues provides a basis for embarking on such clinical vaccine trials in breast cancer patients.

1. van der Bruggen, P., et al. 1991. A gene encoding an antigen recognized by cytotoxic $\mathrm{T}$ lymphocytes on a human melanoma. Science. 254:1643-1647.

2. Knutson, K.L., Schiffman, K., and Disis, M.L. 2001. Immunization with a HER-2/neu helper peptide vaccine generates HER-2/neu CD8 T-cell immunity in cancer patients. J. Clin. Invest. 107:477-484.

3. Mach, N., et al. 2000. Differences in dendritic cells stimulated in vivo by tumors engineered to secrete granulocyte-macrophage colony-stimulating factor or Flt3-ligand. Cancer Res. 60:3239-3246.

4. Jager, E., et al. 1996. Granulocyte-macrophagecolony-stimulating factor enhances immune responses to melanoma-associated peptides in vivo. Int. J. Cancer. 67:54-62.

5. Jaffee, E.M., et al. 2001. Novel allogeneic granulocyte-macrophage colony-stimulating factor-secreting tumor vaccine for pancreatic cancer: a phase I trial of safety and immune activation. J. Clin. Oncol. 19:145-156.

6. Weber, J.S., et al. 2000. Society for Biologic Therapy meeting. November, 2000. Seattle, Washington, USA.

7. Disis, M.L., Grabstein, K.H., Sleath, P.R., and Cheever, M.A. 1999. Generation of immunity to the HER-2/neu oncogenic protein in patients with breast and ovarian cancer using a peptide-based vaccine. Clin. Cancer Res. 5:1289-1297.

8. Disis, M.L., Shiota, F.M., and Cheever, M.A. 1998 Human HER-2/neu protein immunization circumvents tolerance to rat neu: a vaccine strategy for "self" tumour antigens. Immunology. 93:192-199.

9. Dhodapkar, M.V., Krasovsky, J., Steinman, R.M., and Bhardwaj, N. 2000. Mature dendritic cells boost functionally superior $\mathrm{CD} 8\left(^{+}\right)$T-cells in humans without foreign helper epitopes. J. Clin. Invest. 105:R9-R14.

10. Panelli, M.C., et al. 2000. Expansion of tumor-Tcell pairs from fine needle aspirates of melanoma metastases. J. Immunol. 164:495-504.

11. Stewart, J.H., IV, and Rosenberg, S.A. 2000. Longterm survival of anti-tumor lymphocytes generated by vaccination of patients with melanoma with a peptide vaccine. J. Immunother. 23:401-404.

12. Alexander, J., et al. 2000. Linear PADRE T helper epitope and carbohydrate B cell epitope conjugates induce specific high titer IgG antibody responses. J. Immunol. 164:1625-1633.

13. Heathcote, J., et al. 1999. A pilot study of the CY $1899 \mathrm{~T}$-cell vaccine in subjects chronically infected with hepatitis B virus. The CY1899 T Cell Vaccine Study Group. Hepatology. 30:531-536.

14. Candido, K.A., et al. 2001. Local administration of dendritic cells inhibits established breast tumor growth: implications for apoptosis-inducing agents. Cancer Res. 61:228-237.

15. Weber, J.S., et al. 1999. A phase I trial of an HLA-A1 restricted MAGE-3 epitope peptide with incomplete Freund's adjuvant in patients with resected high-risk melanoma. J. Immunother. 22:431-440.

16. Timmerman, J.M., and Levy, R. 2000. Linkage of foreign carrier protein to a self-tumor antigen enhances the immunogenicity of a pulsed dendritic cell vaccine. J. Immunol. 164:4797-4803.

17. Jager, E., et al. 2000. Identification of NY-ESO-1 epitopes presented by human histocompatibility antigen (HLA)-DRB4*0101-0103 and recognized by CD $4\left(^{+}\right) \mathrm{T}$ lymphocytes of patients with NYESO-1-expressing melanoma. J. Exp. Med. 191:625-630.

18. Touloukian, C.E., et al. 2000. Identification of a MHC class II-restricted human gp100 epitope using DR4-IE transgenic mice. J. Immunol. 164:3535-3542.

19. Lee, P.P., et al. 1999. Characterization of circulating $T$ cells specific for tumor-associated antigens in melanoma patients. Nat. Med. 5:677-685

20. Gabrilovich, D.I., Corak, J., Ciernik, I.F. Kavanaugh, D., and Carbone, D.P. 1997. Decreased antigen presentation by dendritic cells in patients with breast cancer. Clin. Cancer Res. 3:483-489. 